\title{
MULTIPLIERS WITH CLOSED RANGE ON REGULAR COMMUTATIVE BANACH ALGEBRAS
}

\author{
PIETRO AIENA AND KJELD B. LAURSEN
}

(Communicated by Palle E. T. Jorgensen)

\begin{abstract}
Conditions equivalent with closure of the range of a multiplier $T$, defined on a commutative semisimple Banach algebra $A$, are studied. A main result is that if $A$ is regular then $T^{2} A$ is closed if and only if $T$ is a product of an idempotent and an invertible. This has as a consequence that if $A$ is also Tauberian then a multiplier with closed range is injective if and only if it is surjective. Several aspects of Fredholm theory and Kato theory are covered. Applications to group algebras are included.
\end{abstract}

\section{INTRODUCTION}

In 1971, at the end of his paper [G], in which the group and measure algebras $L^{1}(G)$, resp. $M(G)$, of a locally compact abelian group $G$ had been considered, Glicksberg points out that his methods of proof really apply to any Tauberian regular commutative semisimple Banach algebra $A$ and its multiplier algebra $M(A)$ (these terms will be defined shortly). Thus his paper establishes the following three results:

(a) If the maximal ideal space $\Delta(A)$ of $A$ is connected, if $A$ satisfies a technical condition (I) (cf. below), and if $T \in M(A)$ is nonzero, then $T A$ is norm closed if and only if $T$ is invertible.

(b) If $A$ satisfies condition (I) and if $T:=T_{a}$ is multiplication on $A$ by the element $a \in A$, then $T A$ is norm closed if and only if $T$ is the product of an idempotent and an invertible multiplier.

(c) If $A$ satisfies condition (I) and if $T \in M(A)$, then $T^{2} A$ is norm closed if and only if $T$ is the product of an idempotent and an invertible multiplier.

The main motivating question of $[\mathrm{G}]$ was whether the factorization of $(b)$ and (c) is necessary for closedness of $T A$, for any multiplier $T$ of $L^{1}(G)$. Its sufficiency is trivial, but the necessity decidedly not. Glicksberg isolated the usefulness of condition (I) in his attempt to give necessary conditions, but not until 1978, in Host and Parreau's impressive paper [HP], was the issue settled, affirmatively, for $L^{1}(G)$. The general question, for a Tauberian regular commutative semisimple Banach algebra $A$, remains open.

In this paper we study this and related questions. Among the main results is Theorem 4.2, which is a partial analog of Host and Parreau's result and which

Received by the editors October 7, 1992.

1991 Mathematics Subject Classification. Primary 47B48, 47C05, 47B06, 46J10, 43A22. 
generalizes a theorem in [DT]; this latter paper studies Segal algebras only. In Theorem 4.2 it is shown, among other things, that if $A$ is a semisimple regular commutative Tauberian Banach algebra and $T \in M(A)$, then $T^{2} A$ is closed if and only if $T$ is the product of an idempotent and an invertible multiplier. This has as a consequence, recorded in Corollary 4.4 , that on a regular semisimple commutative Tauberian Banach algebra a multiplier with closed range will be injective precisely when it is surjective. Other consequences are found in Fredholm theory.

\section{Preliminaries}

We assemble here the definitions of terms most frequently used in the rest of the paper. Even though many of the concepts are perfectly standard, they are included here to facilitate reading.

We shall be considering a commutative Banach algebra $A$, which will always be assumed semisimple. Thus, if $\Delta(A)$ denotes the space of multiplicative linear functionals on $A$, topologized by the weak* topology of the dual space of $A$ and if $a \rightarrow a^{\wedge}$ denotes the Gelfand transform of an element $a \in A$, then this map is injective. The algebra $A$ may be regular, which means that any two disjoint compact and closed, respectively, subsets of $\Delta(A)$ may be separated by elements of $A$. Moreover, if the ideal of $A$, consisting of all elements of $A$ whose Gelfand transforms have compact supports in $\Delta(A)$, is norm dense in $A$, then we call $A$ Tauberian.

If $A$ possesses minimal ideals, the sum of all these is called the socle. If there are no minimal ideals, we set the socle equal to $\{0\}$.

The multiplier algebra $M(A)$ is the commutative subalgebra of the algebra $L(A)$ of all bounded linear operators on $A$, consisting of those operators $T$ for which $a T b=T a b$, for all $a, b \in A$. We shall have occasion to make use of the function $T^{\wedge}$, which here will be the restriction to $\Delta(A)$ of the Gelfand transform of $T$. These notions are more fully described in [L].

One fact about multipliers on semisimple algebras that we shall tacitly use several times is that they have ascent $\leq 1$. In fact, for any linear operator $T$, the ascent $\alpha(T)$ is defined as the smallest nonnegative integer $n$ for which $\operatorname{ker} T^{n}=\operatorname{ker} T^{n+1}$ or $+\infty$ if no such integer exists. If $A$ is a commutative semisimple (semiprime will do, i.e., no nilpotent elements) Banach algebra and $T \in M(A)$, then $\operatorname{ker} T^{2}=\operatorname{ker} T$, because if $T^{2} x=0$ then $0=T^{2} x^{2}=(T x)^{2}$; hence, $T x=0$. An immediate consequence of this is that $T A \cap \operatorname{ker} T=\{0\}$.

The use of notation from Fredholm theory is as follows. The semi-Fredholm operators are, respectively,

$$
\begin{aligned}
& \Phi_{+}(A):=\{T \in L(A) \mid T A \text { is closed and } \operatorname{ker} T \text { is finite dimensional }\}, \\
& \Phi_{-}(A):=\{T \in L(A) \mid T A \text { is of finite codimension in } A\},
\end{aligned}
$$

while the Fredholm operators are $\Phi(A):=\Phi_{+}(A) \cap \Phi_{-}(A)$.

Since $T A \cap \operatorname{ker} T=\{0\}$ for any multiplier $T$ on a semisimple commutative Banach algebra $A$, it is obvious that $\Phi(A) \cap M(A)=\Phi_{-}(A) \cap M(A)$.

Finally, we shall use $\Phi_{0}(A)$ to denote the Fredholm operators with index 0 , i.e., those Fredholm operators $T$ for which $\operatorname{dim} \operatorname{ker} T=\operatorname{codim} T A$, and $\Phi_{M}(A)$ to denote the set of multipliers on $A$ which are invertible modulo the compact multipliers of $A$ (cf., e.g., [A]) . 


\section{The general case: Commutative semisimple Banach algebras}

A recurrent assumption will be that of closedness of $T^{2} A$. The following observations will serve to put perspective on what this means.

Theorem 3.1. If $A$ is a commutative and semisimple Banach algebra and if $T \in M(A)$, then the following conditions are equivalent:

(a) $T^{2} A$ is closed.

(b) $T^{n} A$ is closed, for all $n \in \mathbb{N}$.

(c) The induced multiplier $T^{\sim}: A / \operatorname{ker} T \rightarrow A / \operatorname{ker} T$ has closed range.

(d) $T A+\operatorname{ker} T$ is closed.

Proof. Suppose $T^{2} A$ is closed. Let $B:=T A^{-}$, and note that $B$ is a commutative semisimple Banach algebra with maximal ideal space $\Delta(B)=$ $\Delta(A) \backslash \operatorname{hull}(T A)$. Consider $S:=T \mid B$. Then $S$ is an injective multiplier and has closed range $S(B)=T^{2}(A)$. To see this note first that $S(B) \subseteq T^{2}(A)$, by continuity of $T$ and closedness of $T^{2}(A)$. Since the other inclusion $T(T A) \subseteq$ $T(B)=S(B)$ is obvious, the equality $S(B)=T^{2}(A)$ follows. If $b \in B$ and $S b=0$, then there is a sequence $T a_{n}$ for which $T a_{n} \rightarrow b$ and, hence, for which $T^{2} a_{n} \rightarrow 0$. Since $T^{2}(A)$ is closed, there is a sequence $c_{n}$ in $A$ for which $T^{2} a_{n}=T^{2} c_{n}$ and $c_{n} \rightarrow 0$. But $\operatorname{ker} T^{2}=\operatorname{ker} T$, so $T a_{n}=T c_{n}$; thus, $T c_{n} \rightarrow b=0$.

The Kato resolvent of $S$ is defined to be the set $\rho_{K}(S):=\{\lambda \in \mathbb{C} \mid$ $(S-\lambda) B$ is closed and $\left.\operatorname{ker}(S-\lambda) \subseteq \bigcap_{n \in \mathbb{N}}(S-\lambda)^{n} B\right\}$. Clearly, our assumptions on $S$ imply that $0 \in \rho_{K}(S)$. The inclusion then follows from [S, Satz 4]. In fact, for every $n \in \mathbb{N}, S^{n}(B)$ is closed and an argument like the one just given will show that $S^{n}(B)=T^{n+1}(A)$. This proves that (a) implies $T^{n} A$ is closed for all $n \geq 2$.

Obviously, (b) implies (a).

Let $Q: A \rightarrow A / \operatorname{ker} T$ be the quotient map. Then $T^{\sim}(A / \operatorname{ker} T)=$ $Q(T A+\operatorname{ker} T)$; hence, $Q^{-1}\left(T^{\sim}(A / \operatorname{ker} T)\right)=T A+\operatorname{ker} T$, so $T^{\sim}(A / \operatorname{ker} T)$ is closed if and only if the same holds for $T A+\operatorname{ker} T$. This establishes the equivalence of (c) and (d).

Suppose $A_{0}:=T A+\operatorname{ker} T$ is closed. Let $T A$ be equipped with the norm $|u|:=\inf _{v \in A, u=T v}(\|T v\|+\|v\|)$; in this norm $T A$ is closed. Moreover, since $\|u\| \leq|u|$ for any $u \in T A$, the injection $(T A,\|\|) \rightarrow A_{0}$ is continuous. Define $J: T A \times \operatorname{ker} T \rightarrow A_{0}$ by $J(u, v):=u+v$. Then $J$ is a continuous bijection, so, by the open mapping theorem, $J$ is bicontinuous; hence, $T A=J(T A \times\{0\})$ is closed in $A_{0}$ and, hence, also in $A$.

If $T\left(T a_{n}\right) \rightarrow T a$, then $T\left(T a_{n}-a\right) \rightarrow 0$ and, hence, by openness of $T: A \rightarrow$ $T A$, there is a sequence $\left\{b_{n}\right\}$ in $A$ for which $T b_{n}=T\left(T a_{n}-a\right)$ and $b_{n} \rightarrow 0$. Since $z_{n}:=T a_{n}-a-b_{n} \in \operatorname{ker} T$, it follows that $a+b_{n}=T a_{n}-z_{n} \in T A+\operatorname{ker} T$, hence that $a \in T A+\operatorname{ker} T$, and hence that $T a \in T^{2} A$. It follows that $T^{2} A$ is closed and, hence, that (d) implies (a).

Finally, suppose that $T^{2} A$ is closed, and let $T a_{n}+z_{n} \rightarrow b$, where $z_{n}$ is in $\operatorname{ker} T$. Then $T^{2} a_{n} \rightarrow T b$, so by assumption $T b \in T^{2} A$, i.e., there is $c \in A$ for which $T b=T^{2} c$. Since $z:=b-T c \in \operatorname{ker} T, b=T c+z \in T A+\operatorname{ker} T$ and this shows that $T A+\operatorname{ker} T$ is closed. Consequently, (a) and (d) are equivalent. In particular (a) implies that $T A$ is closed. This completes the proof. 
Adding finiteness assumptions to the assumption of closed range by moving into the classes of semi-Fredholm and Fredholm operators, we may make a couple of observations, to be strengthened later on.

Proposition 3.2. If $A$ is a commutative semisimple Banach algebra, then

$$
\Phi_{M}(A)=\Phi_{0}(A) \cap M(A) .
$$

Moreover, $T \in \Phi_{M}(A)$ if and only if there is a factorization $T=P S$, where $P \in \Phi_{M}(A)$ is an idempotent and $S \in \Phi_{M}(A)$ is invertible.

Proof. If $T \in \Phi_{M}(A)$ then, by commutativity of $M(A)$, there is an element $S \in M(A)$ and a compact multiplier $K \in M(A)$ for which $T S=S T=I+K$. Thus $T S \in \Phi(A)$ and $A=T S A \oplus \operatorname{ker} T S$, since $T S A \cap \operatorname{ker} T S=\{0\}$. It follows that $T S \in \Phi_{0}(A)$ [CPY, Theorem 1.4.7]. Note that $S \in \Phi(A)$, since $S A \supseteq$ $S T A=T S A$. Since the disjointness $W A \cap \operatorname{ker} W=\{0\}$ holds for any $W \in$ $M(A)$, the index of a multiplier is necessarily nonpositive. Consequently, by the index formula ind $T S=$ ind $T+$ ind $S$ [CPY, Theorem 3.2.7]) and ind $T=$ ind $S=0$. This proves $\Phi_{M}(A) \subseteq \Phi_{0}(A) \cap M(A)$.

Conversely, if $T \in \Phi_{0}(A) \cap M(A)$ then $T A$ is a finite codimension and $\operatorname{ker} T$ is a finite-dimensional ideal. By the Wedderburn theorem $\operatorname{ker} T$ is a sum of minimal ideals, so there is an idempotent $e \in A$ for which $\operatorname{ker} T=e A$. Let $L_{e}$ be the multiplication operator defined by $e$. Clearly $T-L_{e} \in \Phi(A) \cap M(A)$ and ind $\left(T-L_{e}\right)=$ ind $T=0$. Moreover, if $x \in \operatorname{ker}\left(T-L_{e}\right)$ then $T x=e x$ so that $T^{2} x=T(e x)=0$, from which, since $\operatorname{ker} T=\operatorname{ker} T^{2}$, we conclude that $x \in \operatorname{ker} T$, i.e., $x=e x=T x=0$. It follows that $\operatorname{ker}\left(T-L_{e}\right)=\{0\}$ and hence that $T-L_{e}$ is invertible. This shows that $T \in \Phi_{M}(A)$.

If $T \in \Phi_{M}(A)$ then the factorization $T=P S$, where $P \in M(A)$ is an idempotent and $S \in M(A)$ is invertible, follows by [A, Theorem 6]. That $S$ belongs to $\Phi_{0}(A)$ is a trivial consequence of invertibility. Also, by the inclusion $P A \supseteq T A$ it is clear that $P$ is an element of $\Phi(A)$ and, hence, of $\Phi_{0}(A) \cap M(A)$, since ind $P$ obviously is 0 .

Conversely, if $T=P S$, where $P \in \Phi_{M}(A)$ is an idempotent and $S \in \Phi_{M}(A)$ is invertible, then, by the index formula [CPY, Theorem 3.2.7], ind $T=$ ind $P+$ ind $S=0$, and hence $T \in \Phi_{M}(A)$.

The ideas of the factorization just introduced may also be used to prove the following generalization of a result of Zaïem [Z, Corollaire, p. 72].

Proposition 3.3. If $A$ is a commutative semisimple Banach algebra with a bounded approximate identity and if $T \in M(A)$, then the following are equivalent.

(a) $T A$ is a closed ideal with a bounded approximate identity.

(b) There is a factorization $T=P S$, where $P \in M(A)$ is an idempotent and $S \in M(A)$ is invertible.

Proof. Let $\left\{e_{\alpha}\right\}$ be a bounded approximate identity of $A$. Suppose $T \in M(A)$ has a factorization $T=P S$, where $P \in M(A)$ is an idempotent and $S \in M(A)$ is invertible. Since $T A=P A, T A$ is a closed ideal. Moreover, the bounded net $\left\{P e_{\alpha}\right\}$ is a subset of $T A$, and, for every $a \in T A, a P e_{\alpha}=P\left(a e_{\alpha}\right) \rightarrow P a=$ $a$. This shows that (b) implies (a).

For the converse, note that if $a \in A$ then, by Cohen factorization applied to $T A$, there are elements $b, c \in A$ for which $T a=T b T c=T(b T c)=T^{2}(b c)$. 
This shows that $T A \subseteq T^{2} A$ and, since the other inclusion is trivial, that $T A=$ $T^{2} A$. Thus $T$ has finite descent, as well as finite ascent, and their common value is $\leq 1$. Hence $A=T A \oplus \operatorname{ker} T$, so, if $P \in M(A)$ is the projection of $A$ onto $T A$, with $\operatorname{ker} P=\operatorname{ker} T$, and if we define $W:=T \mid T A \oplus \operatorname{id}_{\operatorname{ker} T}$, then $W$ is invertible and $T=W P$. Moreover, $W \in M(A)$. Thus we have shown that $T$ is the product of an idempotent and an invertible multiplier.

If we restrict the class of algebras under consideration we can say much more.

\section{ADDING REGULARITY AS AN ASSUMPTION}

The next result will be the main tool in the rest of the paper.

Theorem 4.1. If $A$ is a semisimple regular commutative Banach algebra and if $T \in M(A)$ has the property that $T^{2} A$ is closed, then $T^{\wedge}$ is bounded away from 0 on $\Delta(A) \backslash \operatorname{hull}(T A)$.

Proof. As in the proof of Theorem $3.1,(\mathrm{a}) \Rightarrow(\mathrm{b})$, let $B:=T A^{-}$and note that $B$ is a commutative regular semisimple Banach algebra with maximal ideal space $\Delta(B)=\Delta(A) \backslash \operatorname{hull}(T A)$. Consider $S:=T \mid B$. Then $S$ is an injective multiplier and has closed range $S(B)=T^{2} A$. With the Kato resolvent of $S$ defined as above, our assumptions on $S$ imply that $0 \in \rho_{K}(S)$. In [La, Propositions 1 and 2] it is shown that there is a $\delta_{0} \in \mathbb{R}_{+}$such that for every $\left.\delta \in] 0, \delta_{0}\right]$ the local analytic spectral subspaces $B_{S}\left(\mathbb{C} \backslash D_{\delta}\right)$ are all equal to $\bigcap S^{n}(B)$. Here $D_{\delta}$ is the open disc centered at 0 with radius $\delta$. Now, by [ELN, Proposition 3], this implies that $S^{\wedge^{-1}}\left(D_{\delta}\right)^{-}$are all equal, for every $\left.\delta \in] 0, \delta_{0}\right]$. It follows that $S^{\Lambda^{-1}}\left(D_{\delta_{0}} \backslash\{0\}\right)=\varnothing$. In fact, if $0 \neq \alpha \in D_{\delta_{0}} \cap S^{\wedge}(\Delta(B))$ choose $0<\delta_{1}<|\alpha|<\delta_{0}$; then $\varnothing \neq S^{\wedge^{-1}}(\{\alpha\}) \subseteq$ $S^{\wedge^{-1}}\left(D_{\delta_{0}}\right) \backslash S^{\wedge^{-1}}\left(D_{\delta_{1}}{ }^{-}\right) \subseteq S^{\wedge^{-1}}\left(D_{\delta_{0}}\right) \backslash S^{\wedge^{-1}}\left(D_{\delta_{1}}\right)^{-} \subseteq S^{\wedge^{-1}}\left(D_{\delta_{0}}\right)^{-} \backslash S^{\wedge^{-1}}\left(D_{\delta_{1}}\right)^{-}$-a contradiction. This implies that $S^{\wedge^{-1}}\left(D_{\delta_{0}}\right)=S^{\wedge^{-1}}(\{0\})$ so that $S^{\wedge^{-1}}(\{0\})$ is a clopen set. But then $S^{\wedge^{-1}}(\{0\})=\varnothing$, because otherwise, by regularity of $B$, we may choose a nonzero $a \in B$ for which $\operatorname{supp}\left(a^{\wedge}\right) \subseteq S^{\wedge^{-1}}(\{0\})$. For this $a$ we obtain $(S a)^{\wedge}=0$; hence, $S a=0$, contradicting the injectivity of $S$. Since $S^{\wedge^{-1}}\left(D_{\delta_{2}}\right)=S^{\wedge^{-1}}(\{0\})=\varnothing$, the proposition is proved; we have shown that $\left|S^{\wedge}(\phi)\right| \geq \delta_{0}$ for every $\phi \in \Delta(B)$. Since $S^{\wedge}=T^{\wedge} \mid(\Delta(A) \backslash \operatorname{hull}(T A))$, the proof is complete.

As was noted in Theorem 3.1 for any commutative semisimple Banach algebra $A$, if $T^{2} A$ is closed then so are all the ideals $T^{n} A, n \geq 1$. If $A$ is also Tauberian and regular, these closed ideals are all equal; to see this we show that $T A \oplus \operatorname{ker} T$ is dense in $A$. Since $T A \oplus \operatorname{ker} T$ is closed when $T^{2} A$ is, by Theorem 3.1, this will show that $A=T A \oplus \operatorname{ker} T$. And in this case $T A=T(T A \oplus \operatorname{ker} T)=T^{2} A$, from which an easy induction yields the claim. To show tht $T A \oplus \operatorname{ker} T$, the assumption of Tauberianness means that it suffices to show that $T A \oplus \operatorname{ker} T$ contains every element $x \in A$ with compact support $K:=$ $\operatorname{supp} x^{\wedge}$. Let such $x$ be given and define $x_{1} \in A$ so that $x_{1}^{\wedge}=0$ on $T^{-1}(\{0\})$ and $x_{1}^{\wedge}=x^{\wedge}$ on $K \cap T^{-1}\left(\mathbb{C} \backslash D_{\delta_{0}}\right)$, where $D_{\delta_{0}}$ is as in the previous proof. Also define $x_{2}:=x-x_{1}$. Clearly supp $x_{1}^{\wedge} \subseteq T^{-1}\left(\mathbb{C} \backslash D_{\delta_{0}}\right) \cap K$, so by compactness we conclude from [ELN, Proposition 3] that $\sigma_{T}\left(x_{1}\right) \subseteq T^{\wedge}\left(T^{-1}\left(\mathbb{C} \backslash D_{\delta_{0}}\right)\right) \subseteq \mathbb{C} \backslash D_{\delta_{0}}$, so that $x_{1} \in A_{T}\left(\mathbb{C} \backslash D_{\delta_{0}}\right) \subseteq T A$. On the other hand, supp $x_{2}^{\wedge} \subseteq T^{-1}(\{0\})$, so 
that $\left(T x_{2}\right)^{\wedge}=0$. This shows that $x_{2} \in \operatorname{ker} T$, by semisimplicity and completes the proof.

Thus if $A$ is a regular semisimple commutative Tauberian Banach algebra, if $T \in M(A)$ and if $T^{2}$ has closed range, then $A=T A \oplus \operatorname{ker} T$. Hence $T$ has finite descent, as well as finite ascent, and their common value is $\leq 1$. Hence $A=T A \oplus \operatorname{ker} T$, so, as in the proof of Proposition 3.3, if $P \in \bar{M}(A)$ is the projection of $A$ onto $T A$, with $\operatorname{ker} P=\operatorname{ker} T$, and if we define $W:=T \mid T A \oplus$ $\mathrm{id}_{\text {ker } T}$, then $W$ is invertible and $T=W P$. Moreover, $W \in M(A)$. Thus we have shown that if $T^{2} A$ is closed then $T$ is the product of an idempotent and an invertible multiplier. The converse is clearly true, as well.

This may be phrased slightly differently; since closedness of $T^{2} A$ is equivalent to $T A \oplus \operatorname{ker} T$ being closed, by Theorem 3.1, we see that when $A$ is regular $T A \oplus \operatorname{ker} T$ is closed if and only if $T A \oplus \operatorname{ker} T=A$. We have proved the following result.

Theorem 4.2. If $A$ is a semisimple regular commutative Tauberian Banach algebra and $T \in M(A)$, then the following are equivalent.

(a) $T^{2} A$ is closed.

(b) $T$ is the product of an idempotent and an invertible multiplier.

(c) $T A \oplus \operatorname{ker} T$ is closed.

(d) $T A \oplus \operatorname{ker} T=A$.

If the set $T^{-1}\left(\mathbb{C} \backslash D_{\delta_{0}}\right)$ is compact, as is the case when $T \in M_{0}(A)$, then the condition of Tauberianness is not needed: this time let $x \in A$ be an arbitrary element and define $x_{1} \in A$ so that $x_{1}^{\wedge}=0$ on $T^{-1}(\{0\})$ and $x_{1}^{\wedge}=x^{\wedge}$ on $T^{-1}\left(\mathbb{C} \backslash D_{\delta_{0}}\right)$; this is possible by the regularity of $A$ and the compactness of $T^{-1}\left(\mathbb{C} \backslash D_{\delta_{0}}\right)$. Now proceed exactly as in the above proof of Theorem 4.1 to conclude that if $T^{2} A$ is closed then $A=T A \oplus \operatorname{ker} T$. Thus we obtain a version of Theorem 4.2 for elements of $M_{0}(A)$.

Theorem 4.3. If $A$ is a semisimple regular commutative Banach algebra and $T \in M_{0}(A)$, then the following are equivalent:

(a) $T^{2} A$ is closed.

(b) There is an idempotent $e=e^{2} \in A$ and an invertible multiplier $S$ such that $T=L_{S e}$, the multiplication operator on $A$ defined by $\mathrm{Se}$.

(c) $T A \oplus \operatorname{ker} T$ is closed.

(d) $T A \oplus \operatorname{ker} T=A$.

Proof. (a) $\Rightarrow$ (d) has already been noted before the statement of this theorem, while (a) $\Leftrightarrow$ (c) is Theorem 3.1 .

(a) $\Leftrightarrow$ (b) By Theorem $4.1\left\{\phi \in \Delta(A) \mid T^{\wedge}(\phi) \neq 0\right\}=\left\{\phi \in \Delta(A)|| T^{\wedge}(\phi) \mid>\delta\right\}$ for suitable $\delta \in \mathbb{R}_{+}$. Since $T \in M_{0}(A),\left\{\phi \in \Delta(A) \mid T^{\wedge}(\phi) \neq 0\right\}$ is compact, as well as open. By the Silov theorem there is an idempotent $e \in A$ for which $e^{\wedge}(\phi) \neq 0$ if and only if $T^{\wedge}(\phi) \neq 0$ (if and only if $e^{\wedge}(\phi)=1$ ). Since $(T a)^{\wedge}=(e T a)^{\wedge}$ for any $a \in A$, we conclude that $T a=T e a$, the multiplication operator on $A$ defined by the element $T e \in A$. Next, define $S a:=T e a+a-e a$, for any $a \in A$. Clearly, $S \in M(A)$ and $S e a=e S a=$ $e(T a+a-e a)=e T a=T e a=T a$. Thus only invertibility of $S$ remains unchecked. Letting $\sigma$ denote spectrum we see that $0 \in \sigma(S)$ if and only if there is a sequence $\phi_{n}$ in $\Delta(A)$ for which $(T e-e)^{\wedge}\left(\phi_{n}\right)+1 \rightarrow 0$. Ev- 
idently, this convergence forces that $e^{\wedge}\left(\phi_{n}\right)=1$ for all large $n$, hence that $0 \neq T^{\wedge}\left(\phi_{n}\right) \rightarrow 0$. But if $T^{\wedge}\left(\phi_{n}\right) \neq 0$, then $\left|T^{\wedge}\left(\phi_{n}\right)\right|>\delta$. This proves that $S$ is invertible. For the converse, if $T=L_{S e}$, where $e=e^{2}$ and $S$ is invertible, then $T A=S e A=e S A=e A$, which is closed. This completes the proof.

Another interesting special case of Theorem 4.2 arises when $T \in M(A)$ is injective. We present here a new proof of a result, first noted for Tauberian algebras by Ransford, which in turn was a generalization of the same result for isometric multipliers on Tauberian regular algebras, noted by several authors, e.g., [DT, Theorem 2; ELN, Proposition 8].

Corollary 4.4. If $A$ is a regular semisimple commutative Tauberian Banach algebra, then a multiplier $T \in M(A)$ with closed range is injective if and only if it is surjective.

Proof. Clearly, if $T$ is surjective, then, since $T A \cap \operatorname{ker} T=\{0\}, \operatorname{ker} T=\{0\}$, and hence $T$ is injective.

Conversely, if $T A$ is closed and $\operatorname{ker} T=\{0\}$, then $T A \oplus \operatorname{ker} T$ is closed, so, by Theorem 4.2, $T A=T A \oplus \operatorname{ker} T=A$.

As one of the consequences of Theorem 4.2 we can improve part of Proposition 3.2.

Theorem 4.5. If $A$ is a regular semisimple commutative Banach algebra, then

$$
\begin{aligned}
\Phi_{M}(A) & =\Phi_{0}(A) \cap M(A)=\Phi_{+}(A) \cap M(A) \\
& =\Phi_{-}(A) \cap M(A)=\Phi(A) \cap M(A) .
\end{aligned}
$$

Proof. The equality on the far left follows by Proposition 3.2, while the one on the far right was mentioned among the preliminaries in $\S 2$. To show that $\Phi_{+}(A) \cap M(A)=\Phi_{-}(A) \cap M(A)$, it is enough to show $\Phi_{+}(A) \cap M(A) \subseteq \Phi_{-}(A) \cap$ $M(A)$; the other inclusion is trivial. So let $T \in \Phi_{+}(A) \cap M(A)$. Since $\operatorname{ker} T$ is finite dimensional, $T A \oplus \operatorname{ker} T$ is closed, and hence Theorem 4.2 shows that $A=T A \oplus \operatorname{ker} T$. Thus $T \in \Phi_{-}(A)$. Finally, if $T \in \Phi_{+}(A) \cap M(A)$, the same appeal to Theorem 4.2 will show that $T \in \Phi_{0}(A)$. This completes the proof.

If we combine this result with the second half of Proposition 3.2, we obtain the following.

Corollary 4.6. On a regular semisimple commutative Tauberian Banach algebra every semi-Fredholm multiplier is a product of an idempotent and an invertible multiplier.

Remark. Note that the above theorem and its corollary hold for any semisimple commutative Banach algebra with a dense socle, because such an algebra is regular. Thus Corollary 4.6 generalizes Corollary 7 of [A].

Corollary 4.7. Let $A$ be a regular, semisimple, commutative Banach algebra for which the maximal ideal space $\Delta(A)$ contains no isolated points. If $T \in M(A)$ then $T$ is semi-Fredholm if and only if $T$ is invertible.

Proof. Suppose $T \in M(A)$ is semi-Fredholm. Then $T \in \Phi_{M}(A)$, by Theorem 4.5. Hence $T$ is invertible modulo the compact multipliers. But if $\Delta(A)$ contains no isolated points, then $[K]$ shows that there are no nonzero compact multipliers, and consequently $T$ is invertible. 
With an assumption of connectedness on $\Delta(A)$ Corollary 4.7 allows us to fit the condition of closedness of $T^{2} A$ quite neatly into the equivalence of this result. Compare this to the original Glicksberg claim (a) mentioned in the introduction.

Theorem 4.8. If $A$ is a regular, commutative, semisimple Tauberian Banach algebra with connected maximal ideal space $\Delta(A)$ and if $0 \neq T \in M(A)$, then the following are equivalent.

(a) $T$ is semi-Fredholm.

(b) $T^{2}$ has closed range.

(c) $T$ is invertible.

Proof. If $A$ is finite dimensional then $A=\mathbb{C}$, since $\Delta(A)$ is assumed connected. In this case the theorem is trivially true. So we may assume $A$ infinite dimensional. We know already from Corollary 4.7 that (a) and (c) are equivalent and have to show that (b) implies (c). Suppose $T^{2} A$ is closed. By Theorem 4.2, $A=T A \oplus \operatorname{ker} T$, from which the connectedness assumption and $T \neq 0$ allow us to conclude that $A=T A$. This shows that $T$ is invertible.

We shall now introduce the technical condition (I) used by Glicksberg. This condition has obvious connections to the presence of bounded relative units (in the unitization of $A$ ). It should be noted that, by [Ru, Theorem 2.6.1], condition (I) is satisfied by $L^{1}(G)$, whenever $G$ is a locally compact abelian group.

(I): There is a constant $K \in \mathbb{R}_{+}$with the property that for every $\phi \in \Delta(A)$ and every neighborhood $V$ of $\phi$ there is an element $a \in A$ with Gelfand transform $a^{\wedge}$ supported by $V$ for which $a^{\wedge}(\phi)=1$ and $\|a\| \leq K$.

First a statement analogous to that of Theorem 4.1 -because of condition (I), we can get by with an assumption of closed range for $T$, rather than for $T^{2}$.

Proposition 4.9. Suppose $A$ is a regular, commutative, semisimple Banach algebra which satisfies condition (I). Suppose $T \in M(A)$ is nonzero and has closed range $T A$. Then $T^{\wedge}$ is bounded away from zero on $\Delta(A) \backslash \operatorname{hull}(T A)=\{\phi \in$ $\left.\Delta \mid T^{\wedge}(\phi) \neq 0\right\}$.

Proof. Let $\phi \in \Delta(A) \backslash \operatorname{hull}(T A)$, and pick a compact neighborhood $V$ of $\phi$ so that $V \cap \operatorname{hull}(T A)=\varnothing$. Invoking (I), we may pick an element $c$ such that $\left\{\phi \in \Delta(A) \mid c^{\wedge}(\phi)=0\right\}$ is a neighborhood of $\operatorname{hull}(T A)$ and such that $c^{\wedge}(\phi)=1$, while $\|c\| \leq K$.

By regularity $c \in T A$, so there is an element $b \in A$ for which $T b=c$ and for which $\|b\| \leq C\|c\|$; the latter inequality stems from the assumption of closedness of $T A$, i.e., openness of the map $T: A \rightarrow T A$. We obtain the estimate

$$
1=c^{\wedge}(\phi)=T^{\wedge}(\phi) b^{\wedge}(\phi) \leq\left|T^{\wedge}(\phi)\right|\|b\| \leq\left|T^{\wedge}(\phi)\right| C\|c\| \leq\left|T^{\wedge}(\phi)\right| C K .
$$

Thus $\left|T^{\wedge}(\phi)\right| \geq 1 /(C K)$, and since $\phi \in \Delta(A) \backslash \operatorname{hull}(T A)$ is arbitrary, the proposition follows.

This then gives us a supplement to Theorem 4.3, for multipliers whose transform vanishes at infinity on $\Delta(A)$. This next corollary is a modest improvement of statement $(b)$ of the introduction. 
Corollary 4.10. Suppose $A$ is a regular, commutative semisimple Banach algebra which satisfies condition (I). Let $T \in M_{0}(A)$, so that $T^{\wedge}$ vanishes at infinity on $\Delta(A)$. Then the conditions of Theorem 4.3 are also equivalent with

(e) $T A$ is closed.

Proof. If $T=0$, then everything is trivial. If $T \neq 0$, then, by Theorem 4.9, closedness of $T A$ entails that $T^{\wedge}$ is bounded away from 0 on $\{\phi \in$ $\left.\Delta(A) \mid T^{\wedge}(\phi) \neq 0\right\}$. Now proceed as in the proof of Theorem 4.3, (a) $\Rightarrow(\mathrm{b})$.

\section{GROUP ALGEBRAS}

The most significant applications of the theory developed here are to group and measure algebras of locally compact abelian groups. We note that if $G$ is a locally compact abelian group then $L^{1}(G)$ is a regular semisimple commutative Banach algebra, which satisfies condition (I). Thus we can draw freely on any of the results proved in the previous sections, except Proposition 3.3, which makes an assumption involving approximate identities of ideals. We also take it for granted that the multiplier algebra of $L^{1}(G)$ is the measure algebra $M(G)$, with the elements of $M(G)$ acting as convolution operators on $L^{1}(G)$.

If we collect the results proved, express them in terms of $L^{1}(G)$ and $M(G)$, and take into account Host and Parreau's characterization [HP] of multipliers with closed range, then we have the following.

Theorem 5.1. Let $G$ be a locally compact abelian group, and let $\mu \in M(G)$ be a nonzero regular complex Borel measure on $G$. For the convolution operator $T_{\mu}: L^{1}(G) \rightarrow L^{1}(G)$ consider the following statements.

(a) $\mu$ is invertible in $M(G)$.

(b) $T_{\mu}$ is semi-Fredholm.

(c) $T_{\mu}$ is Fredholm.

(d) $T_{\mu}$ is Fredholm of index 0.

(e) $T_{\mu}$ is invertible modulo the compact multipliers in $M(G)$.

(f) $T_{\mu}$ has closed range.

(g) $T_{\mu * \mu}$ has closed range.

(h) $\mu$ is the product of an idempotent measure and an invertible measure.

Then the following implications hold. (a) implies every other statement on the list. (b), (c), (d), and (e) are equivalent. (f), (g), and (h) are equivalent. The four statements (b)-(e) imply the statements $(\mathrm{f})-(\mathrm{h})$. Moreover, if the dual group $G^{\wedge}$ has no isolated points then the statements (b)-(e) are also equivalent to (a). If the dual group $G^{\wedge}$ is connected, then all the statements $(\mathrm{a})-(\mathrm{h})$ are equivalent.

Proof. That statement (a) implies the others is trivial.

The equivalence of (b)-(e) is a transcription of Theorem 4.5, while Corollary 4.6 shows that $(\mathrm{b})$ implies $(\mathrm{h})$; this implication is also a consequence of Proposition 3.2. By Theorem 4.2, $(\mathrm{g})$ and $(\mathrm{h})$ are equivalent, and the implication $(\mathrm{g})$ $\Rightarrow$ (f) was noted after Theorem 4.1; the implication (f) $\Rightarrow(\mathrm{h})$ is [HP].

If the dual group $G^{\wedge}$ has no isolated points then the equivalence of $(b)-(e)$ with (a) follows from Corollary 4.7.

If $G^{\wedge}$ is connected then Theorem 4.8 finishes the proof. 


\section{REFERENCES}

[A] P. Aiena, Multipliers and projections on semisimple commutative Banach algebras, Proc. Second Internat. Conf. Functional Anal. and Approximation Theory, Rend. Circ. Mat. Palermo 33 (1993), 155-165.

[BMSW] B. A. Barnes, G. J. Murphy, M. R. F. Smyth, and T. T. West, Riesz and Fredholm theory in Banach algebras, Pitman, Boston, MA, 1982.

[CPY] S. R. Caradus, W. E. Pfaffenberger, and B. Yood, Calkin algebras and algebras of operators on Banach spaces, Marcel Dekker, New York, 1974.

[DT] M. Dutta and U. B. Tewari, On multipliers of Segal algebras, Proc. Amer. Math. Soc. 72 (1978), 121-124.

[ELN] J. Eschmeier, K. B. Laursen, and M. M. Neumann, Multipliers with natural local spectra on commutative Banach algebras, submitted.

[G] I. Glicksberg, When is $\mu * L^{1}$ closed?, Trans. Amer. Math. Soc. 160 (1971), 419-425.

[HP] B. Host and F. Parreau, Sur un problème de I. Glicksberg: Les idéaux fermés de type fini de $M(G)$, Ann. Inst. Fourier (Grenoble) 28 (1978), 143-164.

[K] H. Kamowitz, On compact multipliers of Banach algebras, Proc. Amer. Math. Soc. 81 (1981), 79-80.

[L] R. Larsen, Theory of multipliers, Springer-Verlag, Berlin, 1971.

[La] K. B. Laursen, Multipliers and local spectral theory, Banach Center Publ. (to appear).

[LN] K. B. Laursen and M. M. Neumann, Local spectral properties of multipliers on Banach algebras, Arch. Math. 58 (1992), 368-375.

[Ra] T. J. Ransford, private communication.

[Ru] W. Rudin, Fourier analysis on groups, Interscience, New York, 1967.

[S] Ch. Schmoeger, Ein Spektralabbildungssatz, Arch. Math. 55 (1990), 484-489.

[Z] Y. Zaïem, Opérateurs de convolution d'image fermée et unités approchées, Bull. Sci. Math. (2) 99 (1975), 65-74.

Dipartimento di Matematica ed Applicazioni, Università di Palermo, Via Archirafi 34, 90123 Palermo, Italia

E-mail address: aiena@ipamat.cres.it

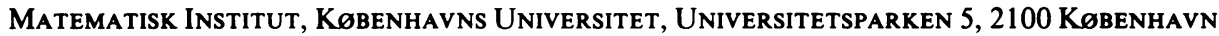
$\emptyset$ DANMARK

E-mail address: laursen@math.ku.dk 